\title{
EUV Airglow Observations by the Satellite TAIYO
}

\author{
Katsuhisa SuzukI \\ Geophysics Research Laboratory, University of Tokyo, Tokyo, Japan
}

(Received September 5, 1978)

\begin{abstract}
An EUV airglow photometer aboard the satellite TAIYO recorded the airglow radiance in the wavelength region of $75-100 \mathrm{~nm}$. The intensity profile of the airglow with respect to altitude measured in daytime and twilight shows good agreement with the model calculation of OII $83.4 \mathrm{~nm}$ emission.
\end{abstract}

\section{Introduction}

An extreme ultraviolet airglow photometer aboard the satellite TAIYO was in operation from March, 1975 through October, 1975. The purpose of the experiment was to measure the extreme ultraviolet emissions of $\mathrm{He}^{+}$at $30.4 \mathrm{~nm}$, $\mathrm{He}$ at $58.4 \mathrm{~nm}, \mathrm{O}^{+}$at $83.4 \mathrm{~nm}$ and $\mathrm{O}$ at $130.0 \mathrm{~nm}$ in the upper atmosphere and geocorona. Recently the terrestrial airglow spectra in the wavelength range below $160 \mathrm{~nm}$ were obtained by spectrometers (CHRISTENSEN, 1976; CARRUTHERS and PAGE, 1976). On the other hand an altitude profile of EUV airglow emissions in the wavelength range $75-105 \mathrm{~nm}$ has been measured by a rocket-borne photometer (CARLSON and JUdGE, 1973).

In this paper we describe the data analysis for the $\mathrm{O}^{+}$sensor aboard TAIYO. Since the emission intensities of the EUV airglow change with the atmospheric condition and solar flux, such observation is an important tool to study thermospheric physics.

\section{Instrumentation}

The detailed description of the instrument is given elsewhere (ToHmatsu et al., 1975). The EUV photometer consists of four optical filters and four channel multipliers. The instrumental specifications are listed in Table 1. The absolute quantum efficiency of the multipliers were calibrated at $\mathrm{H} \mathrm{Ly}-\alpha$ (121.6 $\mathrm{nm})$. The Ly- $\alpha$ flux was measured by a calibrated ion chamber filled with NO gas (OGAWA and Tohmatsu, 1971). The overall sensitivity of the photometer was 0.14 counts $/ R$ at $83.4 \mathrm{~nm}$. The optical axis of the sensor is oriented at $90^{\circ}$ from the satellite spin axis. During one satellite rotation 32 data points are sampled for each sensor. This way 32 directions separated from each other by 
Table 1. The EUV photometer characteristics.

\begin{tabular}{llll}
\hline Solid angle & Area & Filter & Wavelength range \\
\hline 0.0022 ster & $40 \mathrm{~mm}^{2}$ & Indium & $75-100 \mathrm{~nm}$ \\
\hline
\end{tabular}

$11.25^{\circ}$ are recorded. The radiance data of the 32 directions are stored in magnetic core memories aboard the satellite. Since the satellite spin axis was controlled to orient perpendicular to the orbital plane (NinomiYa et al., 1976), the sensor measured the distribution of the radiance along the main circle which contains the earth nadir and local vertical directions. Depending on the altitude of the satellite, the view angle, at which the sensor detects the earth radiance within the main circle was about $90-150^{\circ}$.

The secular variation of the channel multiplier efficiency (SснміDтке et al., 1975) was not taken into account for the present data analysis, because the time period of the data which we analyzed is about 3 months starting about 4 months after the first operation of the experiment.

\section{Observations}

The EUV photometer obtained the data that are analyzed here between July-October, 1975. Figure 1 shows typical data with the satellite orbit. Usually the photometer shows high counting rates with two pronounced peaks at altitudes exceeding $1,500 \mathrm{~km}$. This high background is caused by charged particles from

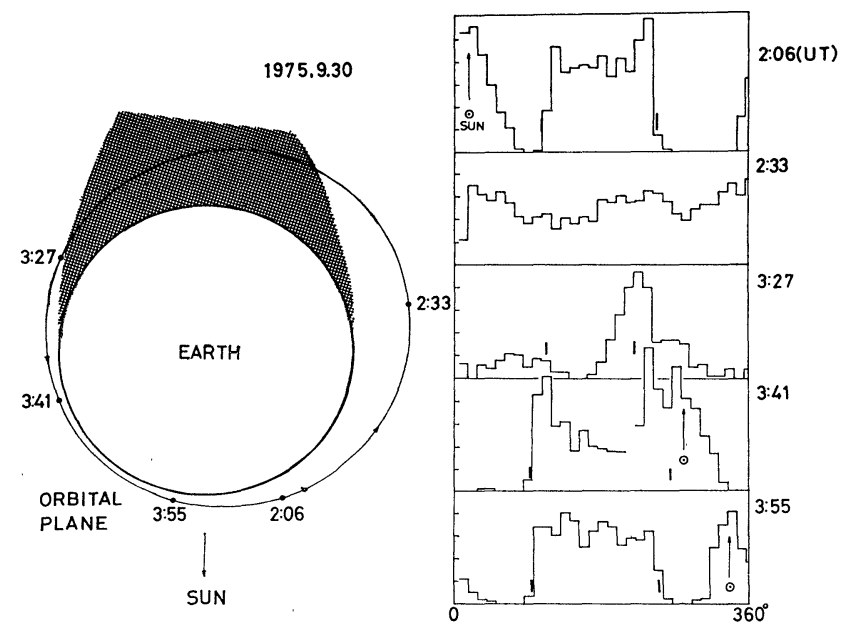

Fig. 1: Typical photometer output. The earth edge is marked by short lines. The satellite positions are represented on the orbit. The dotted region is the earth shadow region. 

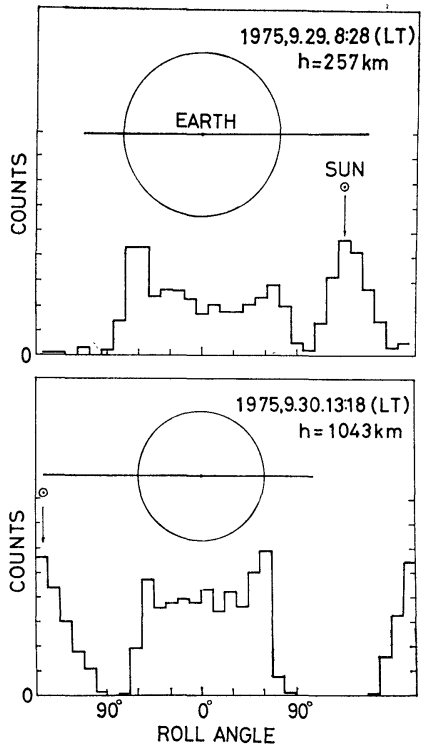

(a)
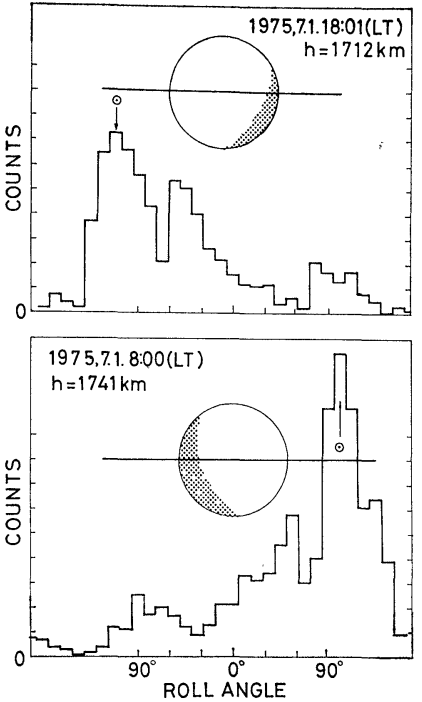

(b)

Fig. 2. (a) The EUV radiance in daytime. (b) The EUV radiance in twilight time. The solar scattering light is detected.

the radiation belt (OGAWA and Tohmatsu, 1971). The SXR instrument aboard the TAIYO satellite also detected high counting rates in the radiation belt. The photometer occasionally was in a position observing the emission from day side to night side across the terminator. The radiance obtained in day side shows limb brightening effect. On the other hand at the twilight condition the intensity decreases gradually across the terminator. In Fig. 2 the radiance profiles in daytime and twilight time are represented.

\section{Discussion and Conclusions}

In the wavelength range of $75-100 \mathrm{~nm}$ emissions from $\mathrm{O}^{+}$at $83.4 \mathrm{~nm}, \mathrm{O}$ at $98.9 \mathrm{~nm}$ and to less extend $\mathrm{N}_{2}$ (Birge-Hopfield bands) are considered the main sources of EUV airglow (CHRISTENSEN, 1976). Taking account of the overall sensitivity of the photometer, the $\mathrm{O}^{+}$emission is considered the most dominant component in the given spectral region. Therefore, we compare the observed data with the $\mathrm{O}^{+}$emission feature for the data analysis. The following excitation processes of the $83.5 \mathrm{~nm}$ line are taken into account:

$$
\begin{aligned}
& \mathrm{O}^{+}+h \nu \rightarrow \mathrm{O}^{+*}, \quad \text { resonance scattering of solar EUV; } \\
& \mathrm{O}+h \nu \rightarrow \mathrm{O}^{+*}+\mathrm{e}, \quad \text { ionization-excitation by photons; }
\end{aligned}
$$




$$
\begin{aligned}
& \mathrm{O}+\mathrm{e} \rightarrow \mathrm{O}^{+*}+2 \mathrm{e}, \quad \text { photoelectron impact; } \\
& \mathrm{O}^{+}+\mathrm{e} \rightarrow \mathrm{O}^{+*}+\mathrm{e}, \quad \text { photoelectron impact. }
\end{aligned}
$$

The process (2) has been studied by Dalgarno and MCElroy $(1965,1966)$. CIRA (1965) atmosphere, the EUV solar flux data (HINTEREGGER et al., 1965) and the O photoionization cross section data (HENRY, 1967) are adopted for the present model calculation. We also used the simplified formula, $\sigma=1.28 \times 10^{-15}$ $f b / \varepsilon W \mathrm{~cm}^{2}$ (Allen, 1973), as the cross section of the process (3) and (4), where $f$ is oscillator strength, $\varepsilon$ the electron energy before collision in Rydbergs, $W$ the excitation energy also in Rydbergs. The photoelectron flux has been calculated by OGAwA (1968). A value of $1.7 \times 10^{-5}$ photons/sec/atom is adopted as the $g$ factor of the process (1).

The production rates of these processes in the case of normal incident solar ray are represented in Fig. 3. As Fig. 3 illustrates, resonance scattering is the dominant component above $250 \mathrm{~km}$ altitude while below $200 \mathrm{~km}$ photoionization-excitation is the main contribution. The photoelectron impact process is rather small compared with the other two processes. The solar EUV resonance scattering by atomic oxygen as another contribution to the EUV radiance in this wavelength range would show the same profile as process (2). The comparison of the model calculation and the observed values at the twilight condition is given in Fig. 4. The calculated values are in good agreement with the observed ones. The gradual decrease of the intensity to the night side can be explained by a decreasing production rate of process (2) at the night side region.

The altitude dependence of the nadir intensity is shown in Fig. 5. In spite

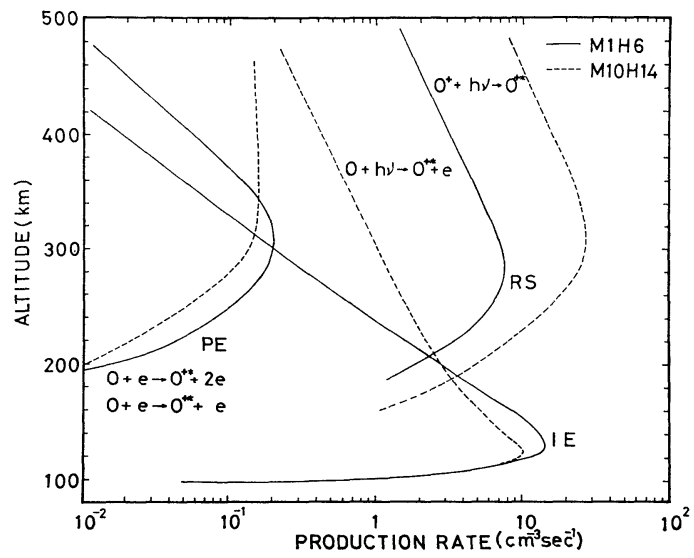

Fig. 3. Altitude profile of the production rate of excited oxygen ion. Calculation is performed for two cases of model $1,6 \mathrm{hr}$ and model 10, 14 hr of CIRA. 

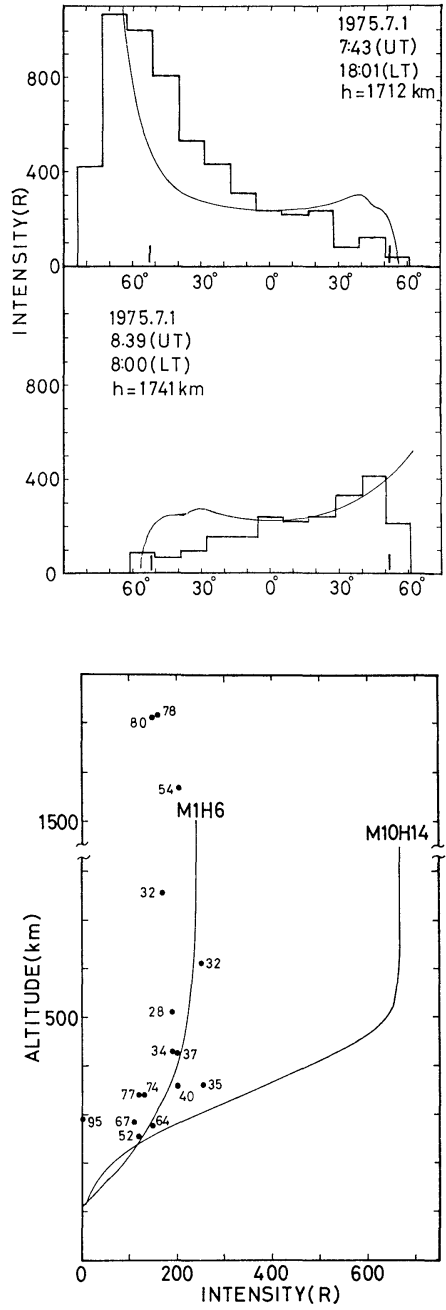

Fig. 4. Comparison of the calculation and the observed values at twilight condition. The observed values are normalized with the nadir intensity of the calculated values.

Fig. 5. The altitude dependence of the nadir intensity. The solar zenith angles are represented by numerals.

of the different observing conditions, it seems that the measured absolute intensity is almost same as the calculated results of CIRA model 1.

The EUV radiance profile with height obtained by the present experiment makes agreement with the model calculation. It is desirable to use the solar flux data observed simultaneously for the model calculation. Fortunately the satellite AEROS observed the solar EUV flux during July, 1974-September, 1975. We will make farther analysis using the AEROS data.

The author wishes to thank Drs. T. Ogawa and G. Schmid tke for their valuable discussions and suggestions. 


\section{REFERENCES}

Allen, C.W., Astrophysical Quantities, p. 42, Univ. of London, The Athlone Press, 1973.

CARLson, R.W. and D.L. JUDGE, Rocket observations of the extreme ultraviolet dayglow, Planet. Space Sci., 21, 879-880, 1973.

Carruthers, G.R. and T. Page, Apollo 16 far ultraviolet spectra of the terrestrial airglow, $J$. Geophys. Res., 81, 1683-1694, 1976.

Christensen, A.B., A rocket measurement of the extreme ultraviolet dayglow, Geophys. Res. Lett., 3, 221-224, 1976.

CIRA 1965 (COSPAR International Reference Atmosphere 1965), North-Holland, Amsterdam, 1965.

Dalgarno, A. and M.B. McElroy, The fluorescence of solar ionizing radiation, Planet. Space Sci., 13, 947-957, 1965.

Dalgarno, A. and M.B. McElroy, Twilight effects of solar ionizing radiation, Planet. Space Sci., 14, 1321-1329, 1966.

Henry, R.J.W., Photoionization cross sections for atomic oxygen, Planet. Space Sci., 15, 1747 $1755,1967$.

HinteregGer, H.E., L.A. HAll, and G. Schmidtke, Solar XUV radiation and neutral particle distribution in July 1963 thermosphere, Space Res., V, 1175-1190, 1965.

Ninomiya, K., E. Hirokawa, K. Shuto, and K. Suzuki, Attitude determination of scientific satellite "Taiyo (1975-014A)," Rept. ISAS, University of Tokyo, Tokyo, Vol. 12, pp. 437476, 1976.

OGAWA, T. Ph.D. Thesis. University of Tokyo, Tokyo, 1968.

OGawa, T. and T. Tohmatsu, Sounding rocket observation of helium 304- and 584-A glow, $J$. Geophys. Res., 76, 6136-6145, 1971.

Schmidtke, G., M. Knothe, and F. Heidinger, Magnetic electron multiplies: Efficiency changes, Appl. Opt., 14, 1645-1648, 1975.

Tohmatsu, T., K. Suzuki, and T. Ogawa, The atmospheric UV instrumentation for the satellite "TAIYO," J. Geomag. Geoelectr., 27, 295-301, 1975. 